\title{
Enhancement of Surface Plasmon Fiber Sensor Sensitivity Through the Grafting of Gold Nanoparticles
}

\author{
Elena MILIUTINA ${ }^{1,2}$, Yevgeniya KALACHYOVA ${ }^{2}$, Pavel POSTNIKOV ${ }^{1,2}$, \\ Vaclav Š́VORĆÍ ${ }^{1}$, and Oleksiy LYUTAKOV ${ }^{1,2^{*}}$ \\ ${ }^{1}$ Department of Solid-State Engineering, Institute of Chemical Technology, Prague 16628, Czech Republic \\ ${ }^{2}$ Department of Technology of Organic Substances and Polymer Materials, Tomsk Polytechnic University, Tomsk \\ 634050, Russia \\ *Corresponding author: Oleksiy LYUTAKOV_～E-mail: lyutakoo@vscht.cz
}

\begin{abstract}
The optical fibers, coated with plasmonic active metal films, represent the simple and unpretentious sensors, potentially useful for measurements of physical or chemical quantities and wide range of analytical application. All fiber-based plasmonic sensors operate on the same physical principle based on changes in the position of the plasmon absorption peak induced by a variation of surrounding medium refractive index. However, the observed spectral differences are often weak, and thus an enhancement of sensor sensitivity is strongly required. In this paper, we propose the immobilization of gold nanoparticles with sharp edges on the thin gold layer, deposited on the multimode fiber surface for improvement of the sensor functionality. The morphological and compositional changes in the gold covered fiber surface were determined by using the atomic force microscopy, scanning electron microscopy, and energy-dispersive X-ray spectroscopy methods. As a result of gold nanoparticles immobilization, the pronounced plasmon energy concentration near the fiber surface occurred, thus enhancing the response of the proposed hybrid plasmonic system to the variation of ambient refractive index. The position of plasmon absorption in the case of the created plasmonic structure was shown to be more sensitive to the changes in the surrounding medium in comparison with the standard sensors based on the bare gold layer.
\end{abstract}

Keywords: Optical fiber; surface plasmon resonance; thin gold film; gold nanoparticles; sensitivity enhancement

Citation: Elena MILIUTINA, Yevgeniya KALACHYOVA, Pavel POSTNIKOV, Vaclav ŠVORČÍK, and Oleksiy LYUTAKOV, "Enhancement of Surface Plasmon Fiber Sensor Sensitivity Through the Grafting of Gold Nanoparticles," Photonic Sensors, 2020, 10(2): 105-112.

\section{Introduction}

Plasmonics and related applications possess a range of advantages in the highly precise detection field [1]. Utilization of surface plasmon resonance provides extremely sensitive detection tool with quintessential applications in life sciences, environmental monitoring, clinical diagnostics, pharmaceutical developments, and food safety [2-4]. The unpretentious sensitivity, achieved with plasmon waves, arises due to the large localization of electromagnetic energy near the metal surface [5-7]. Additionally, compared with another conventional analytical method, including chromatographic and spectrometric techniques, optical based plasmonic sensors are simpler in their 
configuration as well as in the setup crucial for working activity and the data acquisition [8-10].

Especial interesting is the utilization of the plasmon-based sensing technique in a label-free manner $[1,11]$. In this field, the optical fiber sensors based on the surface plasmon resonance, excited in the thin metal film deposited on the top of the optical fiber, provide a range of advantages $[12,13]$. Such structure is sensitive to the refractive index changes of the external environment [14, 15] and can be used as cost-effective and very simple-to-implement alternatives to well established bulky prism configurations, which is often used in the plasmon-based detection/recognition [16, 17]. The fiber optic surface plasmon resonance (SPR) sensors are advantageous for their simple structure, low cost, small sample volume, and remote sensing applications [18, 19]. Detection of $\mathrm{pH}$ [20], temperature [21] and various relevant properties of (bio)chemical compounds [22] was reported with the SPR fiber system.

The construction of fiber optics supporting SPR excitation involves the utilization of single or multimode optical fibers, covered with a metal layer $[23,24]$. For optical reasons, the single-mode based system provides the greatest sensitivity, but the mechanical manipulation with these fibers is complicated due to their high fragility [25]. In contrast, the multimode fibers provide a higher mechanical resistance, but the wavelength position of plasmon resonance is less sensitive to the dielectric environment $[4,8]$. Thus, one of the actual challenges in the field of the SPR based optical sensor system is related to a sensitivity increase, in terms of the shift in the resonance wavelength per unit change in thee refractive indices.

In this paper, we propose a utilization of gold nanoparticles immobilization on the plasmon-active fiber surface, which potentially leads to surface plasmon resonance coupling [26-28], achieved between the thin metal layer and grafted nanoparticles with sharp edges. Such experimental route provides the strong enhancement of plasmon resonance and can enhance the SPR sensor sensitivity. It should also be noted that plasmon supported fiber sensors, whose responses are based on the excitation of surface plasmon resonance on the surface of the thin metal film, are usually considered more sensitive to refractive index changes than localized surface plasmon resonance-based sensors (i.e., metal nanoparticles based sensors) [29, 30]. However, their combination can significantly increase the general structure sensitivity, as demonstrated previously [28].

\section{Experiment}

\subsection{Materials}

Multimode plastic-clad silica optical (PCS) fiber was purchased from CeramOptec (Germany) with core and buffer/cladding diameters of $200 \mu \mathrm{m}$ and $230 / 500 \mu \mathrm{m}$, respectively.

All chemical reagents were used as received without further purification. Chloroauric acid tetrahydrate $\left(\mathrm{HAuCl}_{4} \cdot 4 \mathrm{H}_{2} \mathrm{O}, 99.9 \%\right)$, silver nitrate $\left(\mathrm{AgNO}_{3}, 99.0 \%\right)$, ascorbic acid (AA, 99.0\%), and biphenyl-4,4'-dithiol (BFDT) (95.0 \%) were purchased from Sigma-Aldrich.

\subsection{Samples preparation}

The $1 \mathrm{~cm}$ of PCS fiber shell was thermally removed, and the naked PCS core was purified by using washing with deionized water, acetone, and methanol. A thin film of Au was deposited on the PCS fiber core by sputtering (thickness approx. $40 \mathrm{~nm}$ ). The deposition of $\mathrm{Au}$ was accomplished from Au target (DC Ar plasma; gas purity: 99.995\%; pressure: $4 \mathrm{~Pa}$; discharge power: $7.5 \mathrm{~W}$; sputtering time: $400 \mathrm{~s}$ ). The fibers were fixed with the replaceable SubMiniature version A (SMA) connectors.

AuMs ( $\mathrm{Au}$ multibranched nanoparticles) were prepared according to the procedure published in [31]. Freshly prepared fibers with Au layer were immersed into a methanol solution of BFDT 
$\left(5 \times 10^{-3} \mathrm{~mol} \cdot \mathrm{L}^{-1}\right)$ for $24 \mathrm{~h}$. After incubation, the samples modified by BFDT were washed with methanol and immersed into solutions of AuMs for $24 \mathrm{~h}$. Finally, the samples were cleaned in an ultrasonic bath (in distilled water) and dried under a $\mathrm{N}_{2}$ flow.

\subsection{Measurement techniques}

The scanning electron microscopy (SEM) and energy-dispersive X-ray spectroscopy (EDX) (LYRA3 GMU, Tescan, CR) were used to determine the surface morphology and elemental composition. For quantitative element analysis, the scanning electron microscopy and energy-dispersive X-ray spectroscopy (SEM-EDX) spectra were collected for $20 \mathrm{~min}$ at the operating voltage of $10 \mathrm{kV}$ and a beam current of $600 \mathrm{pA}$.

For sample surface characterization, the peak force atomic force microscopy (AFM) technique was applied. Surface mapping was performed with icon (Bruker) set-up on the areas of $2.5 \times 2.5 \mu \mathrm{m}^{2}$.

Transmission electron microscopy (TEM) images of the AuMs were obtained with a JEOL JEM-1010 instrument (JEOL Ltd., Japan), with an SIS MegaView III digital camera (Soft Imaging Systems, acceleration voltage: $80 \mathrm{kV}$ ), and the analysis was performed by using AnalySIS Software 2.0.

Absorption spectra of the samples were obtained by using an HR2000 (Ocean Optics) spectrometer in the $400 \mathrm{~nm}-1000 \mathrm{~nm}$ wavelength range by using the AvaLight-DHS light source (Avantes).

Raman spectra were measured by using a proRaman-L spectrometer (laser power: $25 \mathrm{~mW}$ ) and a Raman spectrometer with $785 \mathrm{~nm}$ excitation wavelengths. Spectra were measured 30 times, each of them with $3 \mathrm{~s}$ accumulation time.

\subsection{Sensor sensitivity calculation}

The sensor sensitivity was defined by calculating the shift in the resonance wavelength per unit change in refractive index according to the formula
$S_{\lambda n}=\partial \lambda_{\text {res }} / \partial_{n s}(\mathrm{~nm} / \mathrm{RIU})$, where $S_{\lambda n}$ is the SPR fiber sensor sensitivity, $\partial \lambda_{\text {res }}$ is the shift in the SPR resonance wavelength, and $\partial_{n s}$ is the change in the refractive index.

\section{Results and discussion}

In this paper, we propose the immobilization of $\mathrm{Au}$ nanoparticles with sharp edges (so-called multibranched $\mathrm{Au}$ nanoparticles - AuMs) on a thin $\mathrm{Au}$ layer deposited on the surface of the multimode optical fiber. It was expected that the potential plasmonic coupling phenomenon [28], between the thin $\mathrm{Au}$ layer and grafted AuMs, would create the high amplification of the plasmon-related electric field and enhance in this way the sensitivity of the created hybrid structure to the surrounding refractive index. The grafting of AuMs procedure was performed through the dithiol bridges and is schematically presented in Fig. 1. Firstly, the thin $\mathrm{Au}$ film was deposited on the naked optical fiber core and immersed in the solution of biphenyl-4,4'-dithiol (BFDT) to immobilize the organic moieties on the $\mathrm{Au}$ surface. The remained free BFDT thiol groups were further utilized for grafting of AuMs from the solution. As a result, the AuMs were immobilized in the close vicinity to $\mathrm{Au}$ covered fiber core and efficiently interacted with the evanescent plasmon wave, excited by the light propagating through the optical fiber.

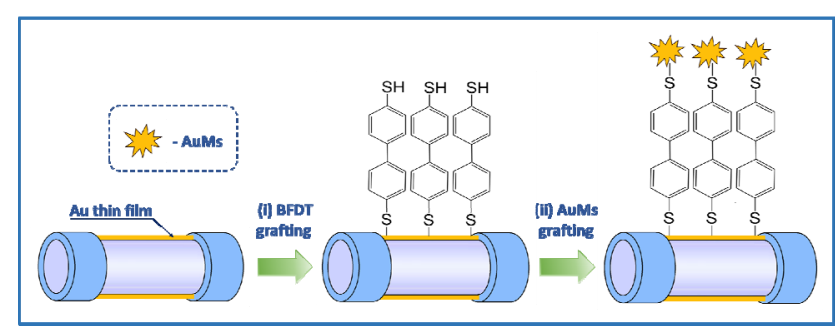

Fig. 1 Schematic representation of the preparation of the hybrid plasmonic sensor based on the plasmon coupling between the thin gold layer deposited on the multimode fiber surface and grafted with (i) dithiol and (ii) AuMs with sharp edges.

The shape and size of AuMs were estimated from TEM images [Fig. 2(a)]. As is evident, the 
AuMs have sharp edges and can effectively contribute to so-called plasmon hot-spots excitation (the places where the local plasmon response was strongly excited). The surface morphology measured by the AFM on the fiber core covered with the $\mathrm{Au}$ layer, before and after AuMs grafting, is presented in Figs. 2(b) and 2(c). The surface of optical fiber covered with the thin $\mathrm{Au}$ film represented a relatively smooth surface, without apparent structural features [Fig. 2(b)]. The significant influence of the grafted AuMs on the surface morphology is evident from Fig. 2(c). AuMs grafting resulted in the appearance of the pronounced surface features and a significant increase in the $R_{a}$ (arithmetical mean deviation from an ideally flat surface) value. The observed differences in the surface roughness after the grafting could be attributed to the non-closed packing of grafted AuMs as well as their sharp edges.

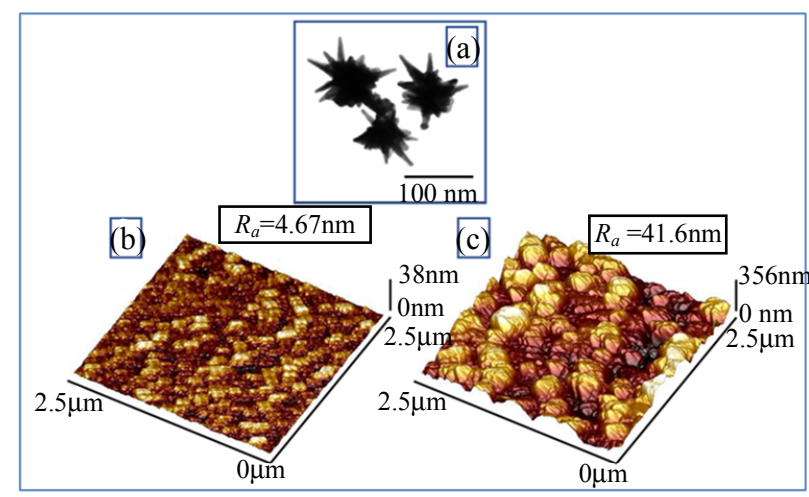

Fig. 2 Characterization of AuMs and fiber surface: (a) TEM image of AuMs nanoparticles with sharp edges; surface morphology of the thin gold film deposited on the multimode fiber core before (b) and after (c) grafting of AuMs.

Additional SEM measurements confirmed the creation of a smooth surface after the deposition of $\mathrm{Au}$ on the fiber core [Fig.3(a)] and conservation of the surface morphology after the BFDT grafting [Fig. 3(b)]. The monomolecular BFDT layer did not affect the surface morphology. A significant increase in the surface topography was observed after the AuMs immobilization on the $\mathrm{Au}$ surface [Fig. 3(c)]. Simultaneously with the SEM, the series of EDX measurements were performed to estimate the surface composition at all stages of samples preparation (see Table 1). The analysis of EDX results confirmed that as-deposited thin $\mathrm{Au}$ layer represented the non-contaminated Au layer. After the BFDT grafting, an increase in the carbon concentration as well as an appearance of sulfur on the fiber surface was observed. Further immobilization of AuMs resulted in a slight decrease in the measured sulfur and carbon concentrations, which was due to the screening effect. The appearance of the Ag-related EDX peak was attributed to the AuMs preparation route.
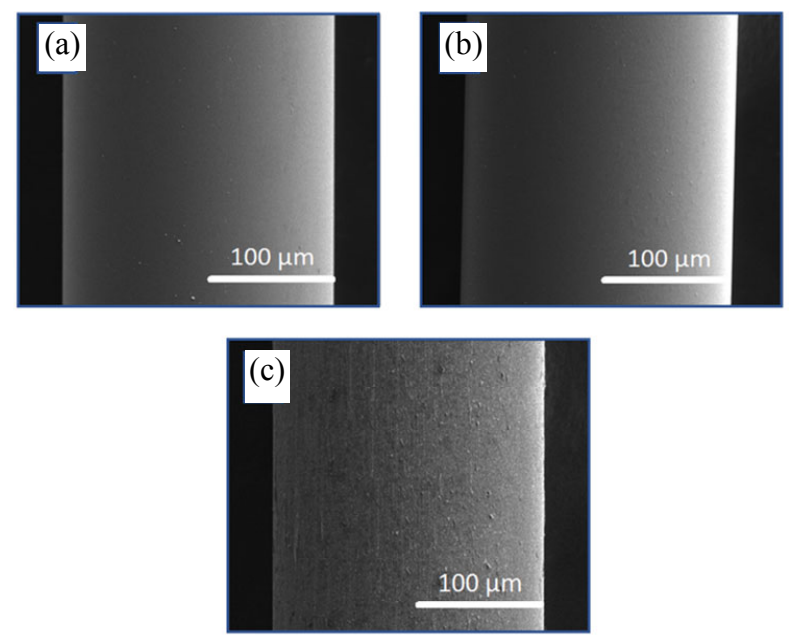

Fig. 3 SEM measured surface morphologies of multimode optical fiber: (a) Au covered fiber surface, (b) after subsequent grafting with BFDT, and (c) grafting of AuMs with sharp edges.

Table 1 Surface elemental composition (measured by SEM-EDX) on the Au coated optical fiber, Au coated optical fiber grafted with BFDT, and Au coated optical fiber with grafted AuMs.

\begin{tabular}{cccc}
\hline \multirow{2}{*}{ Element } & \multicolumn{3}{c}{ Elemental composition (at \%) } \\
\cline { 2 - 4 } & PCS/Au & PCS/Au/BFDT & PCS/Au/BFDT/AuMs \\
\hline $\mathrm{C}$ & 7.61 & 25.51 & 17.44 \\
$\mathrm{O}$ & 2.69 & 2.53 & 3.95 \\
$\mathrm{~S}$ & 0 & 2.86 & 3.1 \\
$\mathrm{Ag}$ & 0 & 0 & 1.94 \\
$\mathrm{Au}$ & 89.7 & 69.1 & 73.57 \\
\hline
\end{tabular}

The BFDT grafting and AuMs immobilization were examined by using the surface-enhanced 
Raman spectroscopy (SERS) measurements (Fig. 4). The pristine Au covered fiber surface did not show any Raman band in the present measurement wavenumber range. Grafting of BFDT resulted in the appearance of weak Raman bands, whose wavenumbers positions corresponded well with the BFDT chemical structure (bands at $1580 \mathrm{~cm}^{-1}$ and $400 \mathrm{~cm}^{-1}$ were attributed to benzene ring, band at $1278 \mathrm{~cm}^{-1}$ corresponded to the stretching of $\mathrm{C}-\mathrm{C}$ between two benzene rings, bands at $1198 \mathrm{~cm}^{-1}$, $1079 \mathrm{~cm}^{-1}$, and $1008 \mathrm{~cm}^{-1}$ represented the $\mathrm{C}-\mathrm{H}$ vibration in benzene ring, and band at $535 \mathrm{~cm}^{-1}$ indicated the presence of the S-S group. The weak intensity of the received Raman signal was obviously due to the formation of the monomolecular organic layer, typical for thiol compounds. Further deposition of AuMs resulted in the significant enhancement of BFDT related SERS signal. This phenomenon confirmed the significant enhancement of electric field intensity between the $\mathrm{Au}$ film and grafted AuMs, which led to a gigantic Raman response from sandwiched BFDT molecules.

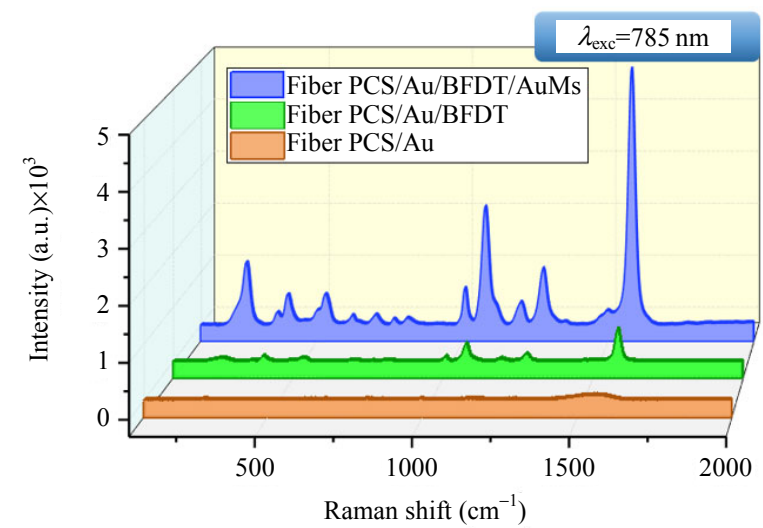

Fig. 4 Proofs of plasmonic coupling - SERS spectra measured on the pristine fibers with bare $\mathrm{Au}, \mathrm{Au}$ surface with grafted with BFDT and Au grafted with BFDT and then with AuMs nanoparticles.

The sensitivity of the created hybrid plasmonic structures to the changes in environmental refractive index was measured in the mixed solution of water/glycerol. The results were compared with the response of $\mathrm{Au}$ optical fiber covered with the bare $\mathrm{Au}$ layer (without grafted AuMs). The normalized results on the light absorption are presented in Figs. 5(a) (fiber covered with the bare Au layer, no AuMs) and 5(b) (fiber covered with the Au layer and grafted with AuMs). The AuMs grafting resulted in the red-shift of plasmon absorption wavelength. Further addition of glycerol resulted in a gradual increase in the surrounding medium refractive index and induced the shift of SPR resonance position in both optical fibers covered with bare $\mathrm{Au}$ and with $\mathrm{Au} / \mathrm{AuMs}$. However, observed spectral changes (i.e. variation of plasmon absorption maximum positions as a function of the surrounding refractive index) were rather different for bare $\mathrm{Au}$ and $\mathrm{Au} / \mathrm{AuMs}$ cases. The SPR fiber sensor with grafted AuMs showed the greatest wavelength shift, indicating an increase in the sensor sensitivity to the changes in the surrounding refractive index.

Figure 6 summarizes the results of SPR measurements and gives the position of the SPR maximum as a function of the surrounding refractive index. In the case of simple SPR fibers, the position of the maximum SPP is shifted from $598 \mathrm{~nm}$ to $707 \mathrm{~nm}$ when the refractive index increases from 1.333 to 1.393. In the case of $\mathrm{Au} / \mathrm{AuMs}$ fibers, the same change in the refractive index leads to the shift of the SPP maximum position from $667 \mathrm{~nm}$ to $860 \mathrm{~nm}$. These results show that the Au/AuMs SPP sensors are two times more sensitive to the changes in the surrounding refractive index.

The present experimental results are summarized in Table2, together with calculated sensor sensitivity values $\left(S_{\lambda n}\right)$. Since the SPR sensor sensitivity is not a constant and can vary with refractive index change, the $\mathrm{S} \lambda \mathrm{n}$ values are given for several measured refractive index intervals. It is evident that the $\mathrm{Au} / \mathrm{AuMs}$ SPR sensor exhibited better sensitivity than the simple SPR sensor with the bare Au layer for all measured refractive index intervals. The higher sensitivity was explained by the strong interaction between the thin $\mathrm{Au}$ layer and grafted AuMs. This phenomenon led to an efficient excitation of plasmon hot spots and an increase in 


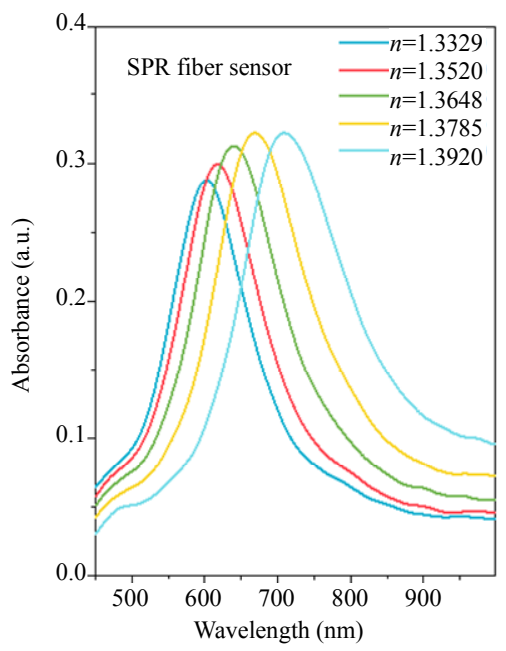

(a)

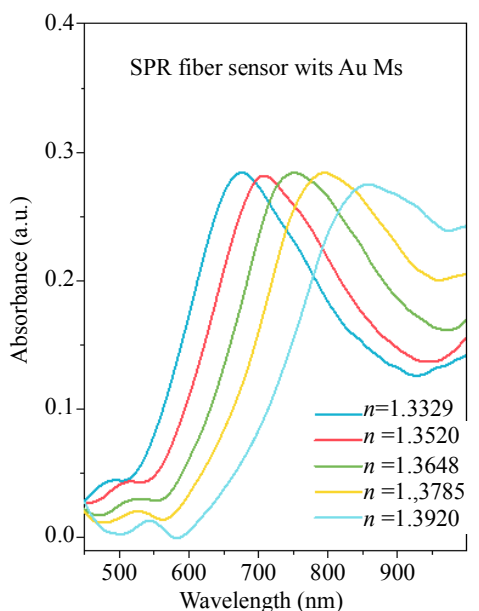

(b)

Fig. 5 Surface plasmon resonance peak as a function of refractive index of surrounding medium (water/glycerol ratio), measured on the: (a) simple SPR sensor and (b) hybrid SPR sensor with grafted AuMs nanoparticles. the SPP sensor sensitivity. The similar phenomenon was observed in the case of SERS enhancement, but to our present knowledge, the utilization of phenomenon for enhancement of SPP fiber optic sensor sensitivity was reported in this work for the first time. In turn, the comparison of obtained results with previously published results indicated that observed enhancement of RIU ( 2 times -3 times, depending on the wavelength range) was similar, where the sensitivity enhancement was reached by using the deposition of the additional layer of silicon or oxide $[32,33]$.

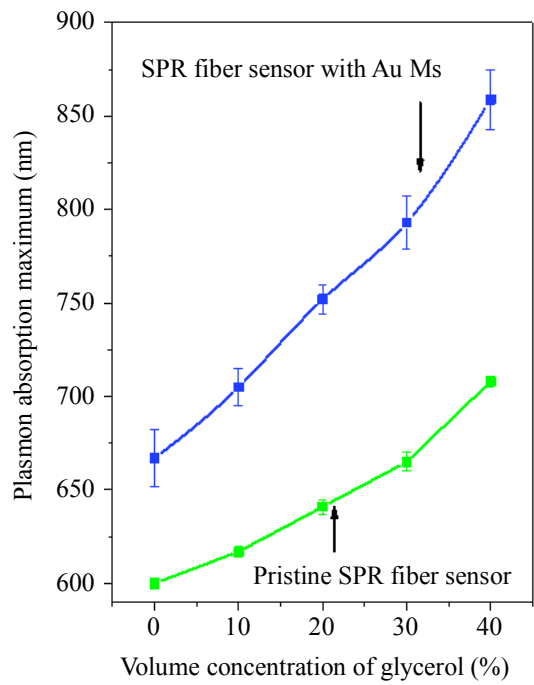

Fig. 6 Dependence of resonance wavelength on volume concentration of water/glycerol for pristine SPR fiber sensor and sensor with grated AuMs.

Table 2 Sensitivity values of plasmon-supported optical fibers with/without grafted AuMs for different refractive indices intervals.

\begin{tabular}{|c|c|c|c|c|c|c|c|}
\hline \multirow{2}{*}{$\begin{array}{l}\text { Refractive indices } \\
\text { (RIU) }\end{array}$} & \multirow{2}{*}{$\begin{array}{c}\Delta n_{s} \\
\text { (RIU) }\end{array}$} & \multicolumn{2}{|c|}{ SPR resonance wavelength } & \multicolumn{2}{|c|}{$\begin{array}{c}\text { SPR sensor sensitivity } \\
S_{\lambda n}(\mathrm{~nm} / \mathrm{RIU})\end{array}$} & \multicolumn{2}{|c|}{$S_{\lambda n} /$ FWHM } \\
\hline & & $\begin{array}{c}\text { Pristine fiber } \\
\text { sensor }\end{array}$ & $\begin{array}{c}\text { Fiber sensor } \\
\text { with AuMs }\end{array}$ & $\begin{array}{c}\text { Pristine fiber } \\
\text { sensor }\end{array}$ & $\begin{array}{c}\text { Fiber sensor } \\
\text { with AuMs }\end{array}$ & $\begin{array}{c}\text { Pristine fiber } \\
\text { sensor }\end{array}$ & $\begin{array}{l}\text { Fiber Sensor } \\
\text { With AuMs }\end{array}$ \\
\hline 1.3329 & & 602 & 672 & & & & \\
\hline 1.3520 & 0.0191 & 614 & 705 & $0.628 \times 10^{3}$ & $1.727 \times 10^{3}$ & 4.94 & 9.18 \\
\hline 1.3648 & 0.0319 & 638 & 752 & $1.128 \times 10^{3}$ & $2.507 \times 10^{3}$ & 8.00 & 11.93 \\
\hline 1.3785 & 0.0456 & 668 & 793 & $1.447 \times 10^{3}$ & $2.653 \times 10^{3}$ & 9.04 & 13.53 \\
\hline 1.3920 & 0.0591 & 710 & 859 & $1.827 \times 10^{3}$ & $3.164 \times 10^{3}$ & 10.81 & 22.12 \\
\hline
\end{tabular}

Finally, we calculated the relation of sensor sensitivity to half-width of the plasmon absorption maximum (since the widening of the absorption band was observed in Fig. 5). Results are also presented in Table 2, and as is evident, slightly worse results were observed in these terms of sensor sensitivity interpretation. Apparently, in this case, the widening of plasmon absorption band took place 
due to the higher distribution of AuMs size and resulted in an insignificant increase in sensor figure of merits after nanoparticles immobilization. This drawback could be further compensated by the utilization of AuMs with narrow distribution (in the case of appearance of the relevant and scalable methods of their preparation) or utilization of narrow light source for probing of refractive index changes.

\section{Acknowledgment}

This work was supported by the (Grant no. P108/12/G108), Tomsk Polytechnic University (Grant no. VIU-RSCABS-196/2018), and the European Structural and Investment Funds, OP RDE-funded project "ChemJets" (Grant no. CZ.02.2.69/0.0/0.0/16_027/0008351).

Open Access This article is distributed under the terms of the Creative Commons Attribution 4.0 International License (http://creativecommons.org/licenses/by/4.0/), which permits unrestricted use, distribution, and reproduction in any medium, provided you give appropriate credit to the original author(s) and the source, provide a link to the Creative Commons license, and indicate if changes were made.

\section{References}

[1] O. Guselnikova, P. Postnikov, M. Erzina, Y. Kalachyova, V. Švorčík, and O. Lyutakov, "Pretreatment-free selective and reproducible SERS-based detection of heavy metal ions on DTPA functionalized plasmonic platform," Sensors and Actuators B: Chemical, 2017, 253: 830838.

[2] S. Liu, Z. Zheng, and X. Li, "Advances in pesticide biosensors: current status challenges and future perspectives," Analytical and Bioanalytical Chemistry, 2013, 405(1): 63-90.

[3] R. C. Stevens, S. D. Soelberg, S. Near, and C. E. Furlong, "Detection of cortisol in saliva with a flow-filtered portable surface plasmon resonance biosensor system," Analytical Chemistry, 2008, 80(17): 6747-6751.

[4] X. Guo, "Surface plasmon resonance based biosensor technique: a review," Journal of Biophotonics, 2012, 5(7): 483-501.

[5] J. N. Anker, W. P. Hall, O. Lyandres, N. C. Shah, J. Zhao, and R. P. Van Duyne, "Biosensing with plasmonic nanosensors," Nature Materials, 2008, 7(6): 442-453.

[6] D. K. Gramotnev and S. I. Bozhevolnyi, "Plasmonics beyond the diffraction limit," Nature Photonics, 2010, 4(2): 83-91.

[7] J. Svanda, Y. Kalachyova, P. Slepicka, V. Svorcik, and O. Lyutakov, "Smart component for switching of plasmon resonance by external electric field," $A C S$ Applied Materials \& Interfaces, 2015, 8(1): 225-231.

[8] C. Caucheteur, T. Guo, and J. Albert, "Review of plasmonic fiber optic biochemical sensors: improving the limit of detection," Analytical and Bioanalytical Chemistry, 2015, 407(14): 3883-3897.

[9] C. Wadell, S. Syrenova, and C. Langhammer, "Plasmonic hydrogen sensing with nanostructured metal hydrides," ACS Nano, 2014, 8(12): 11925-11940.

[10] I. Arghir, F. Delport, D. Spasic, and J. Lammertyn, "Smart design of fiber optic surfaces for improved plasmonic biosensing," New Biotechnology, 2015, 32(5): 473-484.

[11] N. Khansili, G. Rattu, and P. M. Krishna, "Label-free optical biosensors for food and biological sensor applications," Sensors and Actuators B: Chemical, 2018, 265: 35-49.

[12] I. Abdulhalim, M. Zourob, and A. Lakhtakia, "Surface plasmon resonance for biosensing: a mini-review," Electromagnetics 2008, 28(3): 214-242.

[13] R. Slavík and J. Homola, "Ultrahigh resolution long range surface plasmon-based sensor," Sensors and Actuators B: Chemical, 2007, 123(1): 10-12.

[14] Y. Chen, Y. Yu, X. Li, Z. Tan, and Y. Geng, "Experimental comparison of fiber-optic surface plasmon resonance sensors with multi metal layers and single silver or gold layer," Plasmonics, 2015, 10(6): 1801-1808.

[15] J. Homola, "Surface plasmon resonance (SPR)," Analytical and Bioanalytical Chemistry, 2003, 377: 528-539.

[16] W. Hu, Y. Huang, C. Chen, Y. Liu, T. Guo, and B. O. Guan, "Highly sensitive detection of dopamine using a graphene functionalized plasmonic fiber-optic sensor with aptamer conformational amplification," Sensors and Actuators B: Chemical, 2018, 264: 440-447.

[17] V. Voisin, J. Pilate, P. Damman, P. Mégret, and C. Caucheteur, "Highly sensitive detection of molecular interactions with plasmonic optical fiber grating sensors," Biosensors and Bioelectronics, 2014, 51: 249-254.

[18] Q. Liu, Y. Liu, S. Chen, F. Wang, and W. Peng, "A low-cost and portable dual-channel fiber optic surface plasmon resonance system," Sensors, 2017, 17(12): 2797-2804.

[19] B. Lee, J. H. Park, J. Y. Byun, J. H. Kim, and M. G. 
Kim, "An optical fiber-based LSPR aptasensor for simple and rapid in-situ detection of ochratoxin," Biosensors and Bioelectronics, 2018, 102: 504-509.

[20] S. K. Mishra and B. D. Gupta, "Surface plasmon resonance based fiber optic $\mathrm{pH}$ sensor utilizing Ag/ITO/Al/hydrogel layers," Analyst, 2013, 138(9): 2640-2646.

[21] X. Yang, Y. Lu, B. Liu, and J. Yao, "High sensitivity hollow fiber temperature sensor based on surface plasmon resonance and liquid filling," IEEE Photonics Journal, 2018, 10(2): 1-9.

[22] J. F. Masson, L. Obando, S. Beaudoin, and K. Booksh, "Sensitive and real-time fiber-optic-based surface plasmon resonance sensors for myoglobin and cardiac troponin I," Talanta, 2004, 62(5): 865-870.

[23] T. Guo, F. Liu, B. O. Guan, and J. Albert, "Tilted fiber grating mechanical and biochemical sensors," Optics \& Laser Technology, 2016, 78: 19-33.

[24] P. Jia and J. Yang, "Integration of large-area metallic nanohole arrays with multimode optical fibers for surface plasmon resonance sensing," Applied Physics Letters, 2013, 102(24): 243107.

[25] G. Nemova and R. Kashyap, "Fiber-Bragggrating-assisted surface plasmon-polariton sensor," Optics Letters, 2006, 31(14): 2118-2120.

[26] Y. Kalachyova, D. Mares, V. Jerabek, P. Ulbrich, L. Lapcak, V. Svorcik, et al., "Ultrasensitive and reproducible SERS platform of coupled Ag grating with multibranched Au nanoparticles," Physical Chemistry Chemical Physics, 2017, 19(22): 14761-14769.

[27] Y. Kalachyova, D. Mares, V. Jerabek, K. Zaruba, P.
Ulbrich, L. Lapcak, et al., "The effect of silver grating and nanoparticles grafting for LSP-SPP coupling and SERS response intensification," The Journal of Physical Chemistry C, 2016, 120(19): 10569-10577.

[28] T. Maurer, P. M. Adam, and G. Lévêque, "Coupling between plasmonic films and nanostructures: from basics to applications," Nanophotonics, 2015, 4(3): 363-382.

[29] I. Kaminska, T. Maurer, R. Nicolas, M. Renault, T. Lerond, R. Salas-Montiel, et al., "Near-field and far-field sensitivities of LSPR sensors," The Journal of Physical Chemistry C, 2015, 119(17): 9470-9476.

[30] A. Saini, R. Medwal, S. Bedi, B. Mehta, R. Gupta, T. Maurer, et. al., "Axonic $\mathrm{Au}$ tips induced enhancement in Raman spectra and biomolecular sensing," Plasmonics, 2015, 10(3): 617-623.

[31]Y. Kalachyova, A. Olshtrem, O. A. Guselnikova, P. S. Postnikov, R. Elashnikov, P. Ulbrich, et al., "Synthesis characterization and antimicrobial activity of near-IR photoactive functionalized gold multibranched nanoparticles," Chemistryopen, 2017, 6(2): 254-260.

[32] P. Bhatia and B. D. Gupta, "Surface-plasmonresonance-based fiber-optic refractive index sensor: sensitivity enhancement," Applied Optics, 2011, 50(14): 2032-2036.

[33] S. Singh, S. K. Mishra, and B. D. Gupta, "Sensitivity enhancement of a surface plasmon resonance based fibre optic refractive index sensor utilizing an additional layer of oxides," Sensors and Actuators A: Physical, 2013, 193: 136-140. 